\title{
Nearest Keyword Multi-Dimensional Data by Index Hashing
}

\author{
Kavitha Guda \\ Associate Professor \\ Dept. of CSE Hyderabad, TS, India
}

\author{
Doolam Ramdarshan \\ Test Lead \\ Hyderabad, TS, India
}

\begin{abstract}
Catchphrase predicated look for in content prosperous multidimensional datasets encourages various novel applications and executes. In this paper, we consider objects that are marked with catchphrases and are embedded in a vector space. For these datasets, we ponder request that demand the most impervious aggregations of centers slaking a given course of action of watchwords. We propose a novel strategy called ProMiSH (Projection and Multi Scale Hashing) that uses self-confident projection and hash-predicated list structures, and achieves high flexibility and speedup. We present a right and an estimated variation of the count. Our exploratory results on sound and produced datasets show that ProMiSH has up to 60 times of speedup over front line treepredicated frameworks.
\end{abstract}

\section{Keywords}

Clustering, Filtering, Multi-dimensional data, Indexing, Hashing

\section{INTRODUCTION}

Items (e.g., pictures, substance mixes, archives, or specialists in communitarian systems) are regularly described by an accumulation of apropos components, and are ordinarily spoken to as focuses in a multi-dimensional element space. For instance, pictures are spoken to using shading highlight vectors, and customarily have distinct content data (e.g., labels or catchphrases) related with them. In this paper, we consider multi-dimensional datasets where every information point has an arrangement of catchphrases. The nearness of catchphrases in includes space sanctions for the advancement of early actualizes to question and investigate these multidimensional datasets. In this paper, we consider most proximate watchword set (alluded to as NKS) questions on content rich multi-dimensional datasets. A NKS question is an arrangement of utilizer-gave watchwords, and the consequence of the inquiry may incorporate $\mathrm{k}$ sets of information focuses each of which contains all the inquiry catchphrases and structures one of the best $\mathrm{k}$ most impenetrable group in the multi-dimensional space. Fig. 1 shows a NKS question over an arrangement of 2-dimensional information focuses. Each point is labeled with an arrangement of catchphrases. For a question $\mathrm{Q}=\mathrm{fa}$; $\mathrm{b}$; $\mathrm{cg}$, the arrangement of focuses $\mathrm{f} 7 ; 8 ; 9 \mathrm{~g}$ contains all the inquiry watchwords fa; b; cg and shapes the most impenetrable bunch contrasted and some other arrangement of focuses covering all the inquiry catchphrases. Subsequently, the set $\mathrm{f} 7 ; 8$; $9 \mathrm{~g}$ is the main 1 result for the question Q. NKS questions are backup for some applications, for example, photograph partaking in friendly systems, diagram design look, geo location seek in GIS systems1 [1], [2], et cetera. The accompanying is a couple of cases.

1) Consider a photograph sharing pleasant system (e.g., Facebook), where photographs are labeled with individuals names and Fig. 1. A case of a NKS question on a catchphrase labeled multi-dimensional dataset. The main 1 result for inquiry fa; $b$; cg is the arrangement of focuses $\mathrm{f7} ; 8 ; 9 \mathrm{~g}$. areas. These photographs can be implanted in a high dimensional component space of surface, shading, or shape [3], [4]. Here a NKS inquiry can discover a gathering of homogeneous photographs which contains an arrangement of individuals.

2) NKS questions are utilizable for chart design look, where named diagrams are implanted in a high dimensional space (e.g., through Lipschitz inserting [5]) for adaptability. For this situation, a look for a sub chart with an arrangement of assigned marks can be replied by a NKS inquiry in the implanted space [6].

3) NKS questions can also uncover geographic examples GIS can portray an area by a high-dimensional arrangement of properties, for example, weight, sultriness, and soil sorts. In the interim, these districts can also be labeled with data, for example, maladies. A disease transmission specialist can figure NKS inquiries to find designs by finding an arrangement of homogeneous districts with every one of the infections of her advantage. We formally characterize NKS inquiries as takes after. Most proximate Keyword Set. Additionally, a best $\mathrm{k}$ NKS question recovers the best $\mathrm{k}$ competitors with the minimum measurement. In the event that two competitors have break even with widths, at that point they are additionally positioned by their cardinality. Yet subsisting systems using tree-predicated files [2], [7], [8], [9] propose conceivable answers for NKS inquiries on multi-dimensional datasets, the execution of these calculations break down strongly with the incrimination of size or dimensionality in datasets. Our observational outcomes demonstrate that these calculations may take hours to end for a multidimensional dataset of a huge number of focuses. Therefore, there is a purpose for a productive calculation that scales with dataset measurement, and yields pragmatic question effectiveness on sizably voluminous datasets. In this paper, we propose ProMiSH (short for Projection and Multi-Scale Hashing) to empower speedy handling for NKS inquiries. Specifically, we build up a correct ProMiSH (alluded to as ProMiSH-E) that dependably recovers the ideal best $\mathrm{k}$ comes about, and an estimated ProMiSH (alluded to as ProMiSHA) that is more effective as far as time and space, and can get close ideal outcomes by and by. ProMiSH-E uses an arrangement of hash tables and altered files to play out a limited pursuit. The hashing procedure is roused by Locality Sensitive Hashing (LSH) [10], which is a cutting edge strategy for most proximate neighbor look in high-dimensional spaces. Not at all like LSHpredicated strategies that authorize just inexact inquiry with probabilistic ensures, the file structure in ProMiSH- 
E sustains exact pursuit. ProMiSH-E induces hash tables at various canister widths, called list levels. A solitary round of hunt in a hash table yields subsets of focuses that contain inquiry results, and ProMiSH-E investigates every subset using a quick pruning-predicated calculation. ProMiSH-An is an inexact variety of ProMiSH-E for better time and space proficiency. We assess the execution of ProMiSH on both bona fide and manufactured datasets and utilize best in class VbR-Tree [2] and CoSKQ [8] as baselines. The observational outcomes uncover that ProMiSH reliably beats the pattern calculations with up to 60 times of speedup, and ProMiSH-An is up to 16 times more quick than ProMiSH-E acquiring close ideal outcomes.

\section{RELEGATED WORK}

\subsection{Existing System}

Existing systems using tree-predicated lists propose conceivable answers for NKS questions on multi-dimensional datasets, the execution of these calculations weakens forcefully with the incrementation of size or dimensionality in datasets. Our experimental outcomes demonstrate that these calculations may take hours to end for a multi-dimensional dataset of a large number of focuses. subsisting works primarily focus on the kind of inquiries where the directions of question focuses are kenned. But it is conceivable to make their cost capacities same to the cost work in NKS questions, such tuning does not transmute their procedures. In the mean time, most proximate neighbor questions ordinarily require organize data for inquiries, which makes it laborious to build up a productive strategy to tackle NKS questions by subsisting procedures for most proximate neighbor seek.

\subsection{Proposed System}

We propose ProMiSH (short for Projection and Multi-Scale Hashing) to empower quick handling for NKS inquiries. Specifically, we build up a correct ProMiSH (alluded to as ProMiSH-E) that dependably recovers the ideal best $\mathrm{k}$ comes about and a rough ProMiSH (alluded to as ProMiSHA) that is more effective as far as time and space, and can acquire close ideal outcomes by and by. The proposed systems utilize area data as an essential part to play out a best-first hunt on the IRTree, and inquiry organizes assume a key part in for all intents and purposes each progression of the calculations to prune the pursuit space. proposed answers for the predicament of best $\mathrm{k}$ most proximate watchword set inquiry in multi-dimensional datasets. We proposed a novel list called ProMiSH predicated on discretionary projections and hashing. Predicated on this file, we created ProMiSH-E that finds an ideal subset of focuses and ProMiSH-A that tests close ideal outcomes with better proficiency.

\section{IMPLEMENTATION \\ 3.1 Multi-dimensional Data}

Catchphrase predicated look in content rich multi-dimensional datasets encourages numerous novel applications and executes. Multi-dimensional datasets where every information point has an arrangement of catch phrases. The nearness of watchwords in highlight space sanctions for the improvement of early actualizes to inquiry and investigates these multidimensional datasets. These calculations may take hours to end for a multi-dimensional dataset of a huge number of focuses. Thus, there is an aim for a productive calculation that scales with dataset measurement, and yields commonsense inquiry effectiveness on sizably voluminous datasets. Multidimensional spaces, it is exhausting for clients to give foremost arrange, and our work manages another kind of inquiries where clients can just give watchwords as information.

\subsection{Most proximate Keyword}

We consider multi-dimensional datasets where every information point has an arrangement of catchphrases. The nearness of catchphrases in include space sanctions for the improvement of early executes to inquiry and investigate these multi-dimensional datasets. A NKS inquiry is an arrangement of utilizer-gave catchphrases, and the aftereffect of the question may incorporate $\mathrm{k}$ sets of information focuses each of which contains all the question watchwords and structures one of the best $\mathrm{k}$ most impenetrable group in the multi-dimensional space. Area straight out watchword questions on the web and in the GIS frameworks were prior addressed using an amalgamation of R-Tree and altered record. Created IR2-Tree to rank articles from spatial datasets predicated on a cumulation of their separations to the question areas and the relevance of their content depictions to the inquiry watchwords.

\subsection{Ordering}

Ordering time as the measurements to assess the record estimate for ProMiSH-E and ProMiSH-A. Ordering time indicates the term used to assemble ProMiSH variations. the memory usage and ordering time of ProMiSH-E and ProMiSH-An under various information valid information. Memory usage develops slowly in both ProMiSH-E and ProMiSH-A when the quantity of measurements in information focuses increments. ProMiSH-An is more effective than ProMiSH-E as far as memory usage and ordering time: it takes $80 \%$ less memory and $90 \%$ less time, and can acquire close ideal outcomes.

\subsection{Hashing}

The hashing system is motivated by Locality Sensitive Hashing (LSH), which is a cutting edge strategy for most proximate neighbor seeks in high-dimensional spaces. Not at all like LSH-predicated strategies that authorize had just rough hunt with have probabilistic ensured, the record structure in ProMiSH-E braces exact inquiry. Erratic projection with hashing has come to be the best in class strategy for most proximate neighbor seek in highdimensional datasets.

\section{EXPERIMENTAL RESULTS}

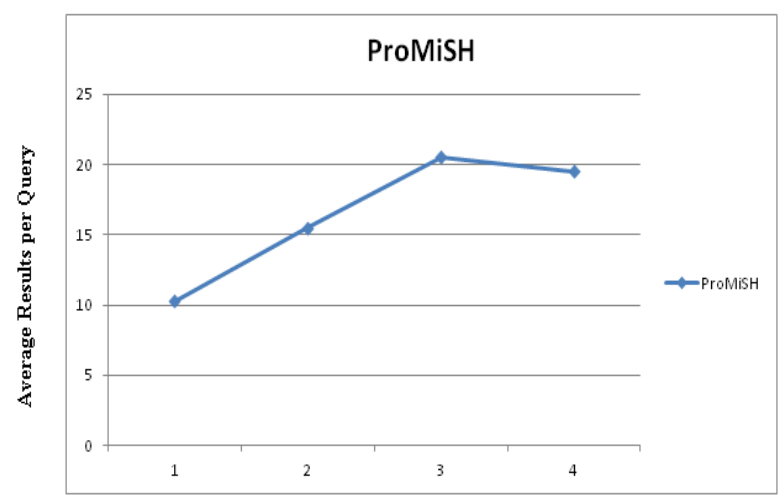

Keywords per Query 


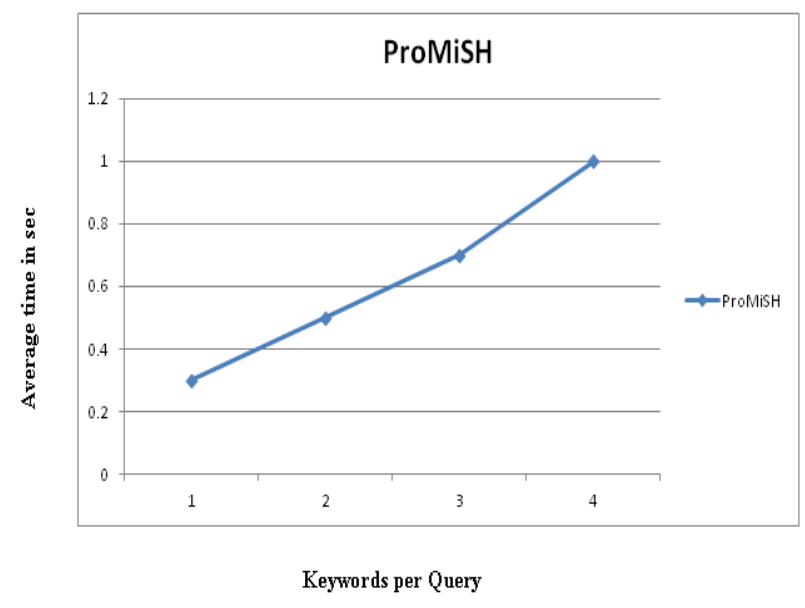

The experiment programs are coded using the JAVA programming language on a Laptop with $2.2 \mathrm{GHZ}$ Intel Core CPU and GB memory. We use the ProMiSH [64] for data encryption. We found the two graphs with average time cost of no of queries.

\section{CONCLUSION}

In this paper, we proposed answers for the pickle of best $\mathrm{k}$ most proximate catchphrase set hunt in multi-dimensional datasets. We proposed a novel list called ProMiSH predicated on erratic projections and hashing. Predicated on this list, we created ProMiSH-E that finds an ideal subset of focuses and ProMiSH-A that tests close ideal outcomes with better proficiency. Our experimental outcomes demonstrate that ProMiSH is more speedy than cutting edge tree-predicated methods, with numerous requests of size execution revision. Besides, our methods scale well with both true and engineered datasets. Positioning capacities. Later on, we organize to investigate other scoring plans for positioning the outcome sets. In one plan, we may allocate weights to the watchwords of a point by using strategies like tf-idf. At that point, each gathering of focuses can be scored predicated on remove amongst focuses and weights of watchwords. Besides, the criteria of an outcome containing every one of the catchphrases can be casual to cause comes about having just a subset of the inquiry watchwords. Circle expansion. We arrange to investigate the expansion of ProMiSH to plate. ProMiSH-E successively peruses just required basins from Ikp to discover focuses containing no less than one inquiry catchphrase. Therefore, Ikp can be put away on plate using a registry document structure. We can cause a registry for Ikp. Each can of it will be put away in a different record assigned after its key in the index. In addition, ProMiSH-E consecutively tests $\mathrm{HI}$ information structures beginning and no more little scale to induce the competitor point ids for the subset pursuit, and it peruses just required containers from the hash table and the reversed record of a HI structure. Subsequently, all the hash tables and the rearranged lists of HI can again be put away using a homogeneous registry document structure as Ikp, and every one of the focuses in the dataset can be ordered into a B+-Tree using their ids and put away on the plate. Along these lines, subset pursuit can recover the focuses from the circle using $\mathrm{B}+$-Tree for investigating the last arrangement of results

\section{REFERENCES}

[1] W. Li and C. X. Chen, "Efficient data modeling and querying system for multi-dimensional spatial data," in GIS, 2008, pp. 58:1-58:4.

[2] D. Zhang, B. C. Ooi, and A. K. H. Tung, "Locating mapped resources in web 2.0," in ICDE, 2010, pp. 521532.

[3] V. Singh, S. Venkatesha, and A. K. Singh, "Geoclustering of images with missing geotags," in GRC, 2010, pp. 420-425.

[4] V. Singh, A. Bhattacharya, and A. K. Singh, "Querying spatial patterns," in EDBT, 2010, pp. 418-429.

[5] J. Bourgain, "On lipschitz embedding of finite metric spaces in Hilbert space," Israel J. Math., vol. 52, pp. 46$52,1985$.

[6] H. He and A. K. Singh, "Graphrank: Statistical modeling and mining of significant subgraphs in the feature space," in ICDM, 2006, pp. 885-890.

[7] X. Cao, G. Cong, C. S. Jensen, and B. C. Ooi, "Collective spatial keyword querying," in SIGMOD, 2011.

[8] C. Long, R. C.-W. Wong, K. Wang, and A. W.-C. Fu, "Collective spatial keyword queries: a distance ownerdriven approach," in SIGMOD, 2013.

[9] D. Zhang, Y. M. Chee, A. Mondal, A. K. H. Tung, and M. Kitsuregawa, "Keyword search in spatial databases: Towards searching by document," in ICDE, 2009, pp. 688-699.

[10] M. Datar, N. Immorlica, P. Indyk, and V. S. Mirrokni, "Locality-sensitive hashing scheme based on p-stable distributions," in SCG, 2004. 